\title{
Image-Based Control of Interactive Robotics Systems
}

\author{
Andreas Hein and Tim C. Lueth \\ Surgical Robotics Lab (SRL) \\ Clinic for Maxillofacial Surgery, Charité Virchow-Hospital, D-13353 Berlin, Germany \\ \{a.hein, t.lueth\}@ieee.org \\ http://www.charite.de/rv/mkg/srl/index.html
}

\begin{abstract}
In this paper a robotics system is described which supports the surgeon during drilling and shaping operations. The special feature of this system is the fusion of control commands by the surgeon and control commands derived directly in real-time from the image data of the patient. This interactive control of a robotics system leads to a cooperation between the surgeon and the robotics system. Because the robotics system can directly access to the image data within every control cycle it provides a higher accuracy and an enhanced safety of drilling operations. A tool for the quantitative analysis of the image data, the interactive control system of the robotics system and the first application in Anaplastology is presented.
\end{abstract}

\section{Introduction}

Surgery is a relatively new and a rapidly growing field of application for robotics technology. Robots can be used to enhance the accuracy and the dexterity of a surgeon, can decrease the tremble of the human hand and can amplify or reduce the movements and/or forces applied by the surgeon. Especially in fields of surgery where the human hand is the limiting factor for further optimization of the surgical techniques -like in neurosurgery, orthopedic and maxillofacial surgery- robotics technology can be applied.

The drilling or shaping of bone structures is of great importance for surgery. In contrary to soft tissue a static model of bone structures derived from CT images can be used. Currently, these operations are carried out by the surgeons free hand during an intervention. In the near of sensitive regions the manual handling can lead to complications due to inaccuracies or shattering of the drill or shaper.

Especially in maxillofacial surgery the accuracy of an intervention is of paramount importance due to the high social and aesthetic impact of the face. Therefore, the positioning and moving of drills or shapers with a high accuracy is desirable. Additional difficulties in maxillofacial surgery are the restricted access to the bone structures through small incisions, the swelling of tissue during the intervention, and the small distance to vital organs or structures. 
This paper presents the image-based control of the first interactive robotics system developed for the application in maxillofacial surgery at the Surgical Robotics Lab (SRL) at the Virchow hospital (Fig. 1). The concept of the semi-active robot controlling will be refined and the interactive use of the robot based on different information entities will be explored.

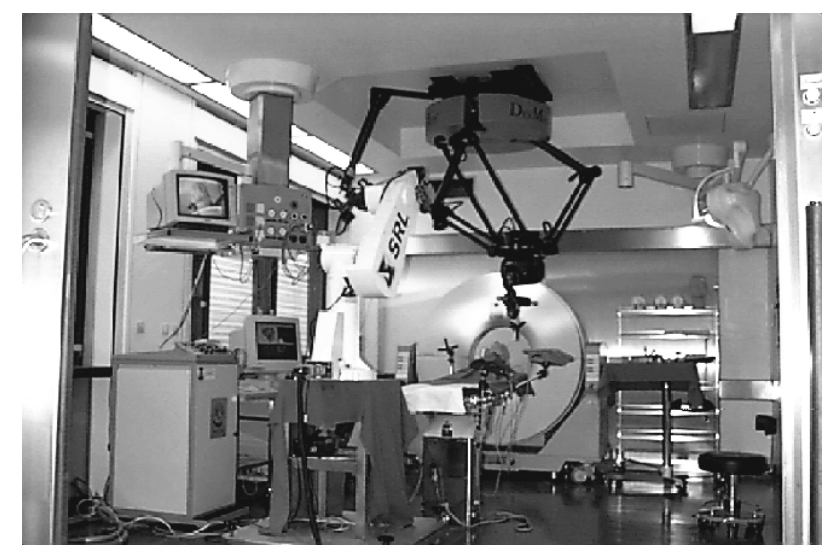

Fig. 1: Experimental OR at the Virchow hospital

\section{State of the Art}

During the last 10 years, robotics techniques have been introduced to operating theaters for only a few surgical applications. The first systems were used in neurosurgery for the guidance of surgical instruments like biopsy needles, catheters, or microscopes $[1,2,3,4]$. After an initial registration of the patient (determination of the transformation matrix between the image data of the patient, the patient at the intraoperativ position and the robot base frame [5]) the manipulators are used to approach points planned in the preoperative image data. During the treatment the manipulators are either switched off or the brakes are locked.

Active robotics systems are used in orthopedic surgery and for laparoscopic applications. The Robodoc system has been developed for the milling of holes exactly fitting for a hip prosthesis [6]. This system works fully automatic during the operation. That means, the robot executes exactly the preoperative planned movements and can react to environmental changes only with an interruption of the program.

The only interactively controllable robotics system for invasive application has been designed for knee surgery [7]. This experimental 2-link arm is force controlled and can be guided by the surgeon manually to the cutting position at the knee bone. The workspace of the cutter is restricted by decreasing controller gains in the near of the boundaries.

The most successful commercial robotics system is the AESOP system by Computer Motion. This camera holding and positioning system has been designed for laparo- 
scopic surgery and has been used in over 30,000 cases [8]. These systems can be interactively used during the operation like the microscope holding systems SurgiScope by Elekta and the MKM by Zeiss.

In addition to the author's system [12], in maxillofacial surgery only two systems are under development. The system described in [9] consists of a passive manipulator used for positioning instruments. The disadvantage of passive systems is the time consuming positioning procedure and the impossibility to move along desired trajectories with force constrains. The other system [10] is an experimental automatic system that is not designed for interactions with the surgeon. Such systems seem not to be suitable for real applications in maxillofacial surgery.

\section{Description of the Treatment}

To approach the complex field of this surgery and to collect first experiences in drilling and shaping operations the placement of implants for the fixation of an extraoral ear epithesis has been selected as the first application of the robotics system at the ${ }^{\circ}$ SRL.

The medical indication for ear epithesis is given in the case of the resection of the ear due to a tumor resection or the destruction of the ear due to an accident. If a reconstruction fails, an artificial ear can either be modeled using the mirror-image of the CT images of the other side's ear or by a manual procedure using impressions. Fig. 2 shows a scheme for the determination of the optimal implant positions around the center of the ear. The implant manufacturer suggests that the implants should be placed on a circle with a diameter of about $2 \mathrm{~cm}$ around the ear channel within the marked areas.

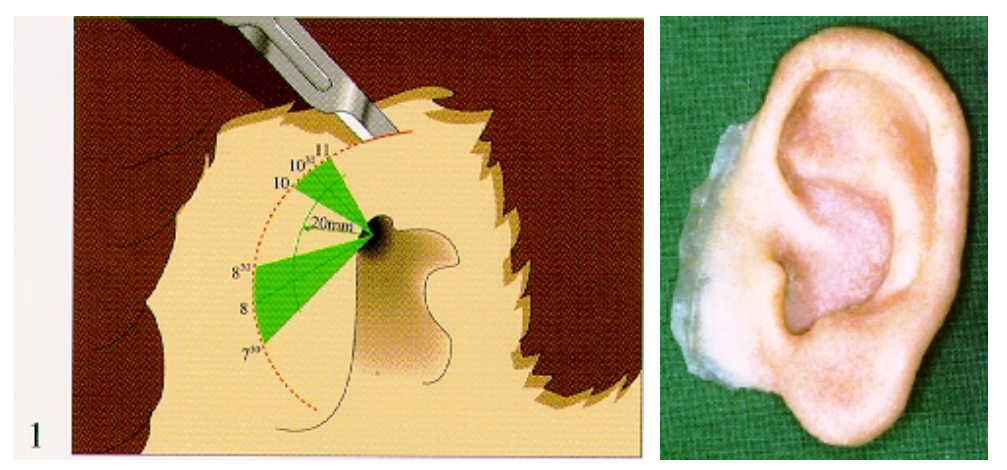

Fig. 2: Planning of the implants' position and placing of the implants [11] (left) and an artificial ear (right)

Using a robotics system for the drilling of the holes and the placement of the implants decreases the number of needed implants to two instead of three. This is caused by the higher stability of the implants. The stability is increased due to a better analysis of the bone structure and due to the exact placement of the implants at the pre-planned posi- 
tions. Especially, the avoidance of hitting mastoid cells (air-filled cavities in the near of the central ear) increases the stability and decreases the risk of infections. To fix the artificial ear a superconstruction has to be mounted at the implants. An artificial ear -as shown on the right side of Fig. 2- will be clipped onto the superconstruction. The construction consists of distance pieces and a bridge between the distant pieces.

The preoperatively known positions of the implant and the superconstruction allow the fabrication of the ear epithesis before the treatment and supply the patient with the ear right after the healing phase. Currently, the patient must wait months for the epithesis because the implant positions are determined after the interventions by impressions.

\section{Architecture of the Robot Controller}

The SurgiScope by Elekta is used as the basis for the development of the robotics system for maxillofacial surgery. This system is originally aimed at the carriage of a microscope for neurosurgery. For invasive applications the controller software and the tools have been changed [12]. The robotics system consists of a parallel manipulator, an infrared navigation system, a control cabinet with the computer for the navigation system and the control computer of the manipulator, and the drilling machine. The drilling machine consists of a drilling station and the hand piece that is mounted to the manipulator.

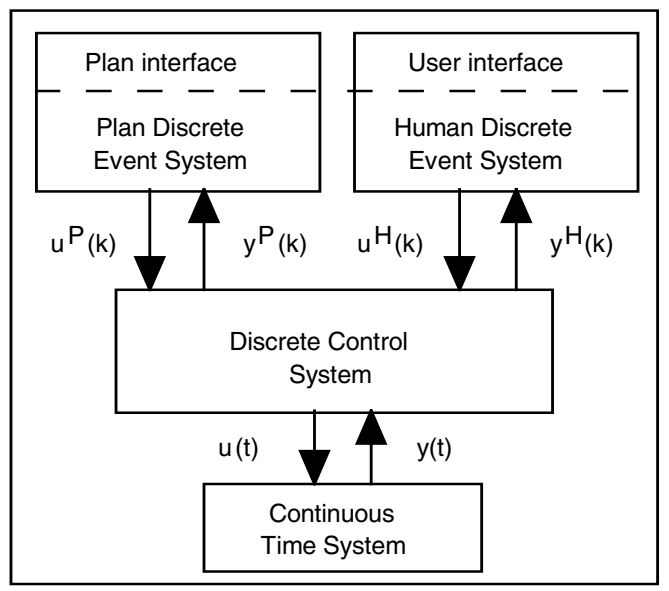

Fig. 3: Architecture of the interactive robot controller [13]

The hand piece of the drilling machine is an unmodified standard surgical hand piece that is mounted to the manipulator by a special construction. Its motor is connected to a station that controls the speed and the force of the drilling machine.

From the perspective of a robot control system which has two distinct information sources this system can be divided into a Human Discrete Event System (HDES) with the user interface, a Plan Discrete Event System (PDES) with the plan interface, a 
Discrete Control System (DCS), and a Continuous Time System (CTS). Fig. 3 shows an overview of the system.

The PDES is a finite state machine, which encodes the general command sequence for a type of intervention. Additionally, it contains specific patient data as target positions ${ }^{I} \boldsymbol{t}$ and registration points ${ }^{I} \boldsymbol{p}$ in respect to the image data coordinate system, and paths between working points outside the patient model. Depending on the current state $s^{P}$ of the PDCS an elementary operation $\boldsymbol{o}(k)$ in the DCS will be activated by the command

$$
\boldsymbol{u}^{P}(k)=\left(\boldsymbol{o}(k),{ }^{I} \boldsymbol{t},{ }^{I} \boldsymbol{p}, \text { path }\right)
$$

Each elementary operation terminates by setting one condition out of the vector $^{\circ} \boldsymbol{y}^{P}(k)$ true and then the PDCS switches to the next state, which corresponds to the true condition. Depending on the active elementary operation $\boldsymbol{o}(k)$ and the current position and velocity of the TCP in respect to the image data coordinate system

$$
\boldsymbol{y}^{H}(k)=\left(\boldsymbol{o}(k),{ }^{I} \boldsymbol{p}_{T C P},{ }^{I} \dot{\boldsymbol{p}}_{T C P}\right)
$$

the HDES generates a command

$$
\boldsymbol{u}^{H}(k)=\left({ }^{I} \dot{\boldsymbol{p}}_{T C P}\right)
$$

that will be delivered to the DCS.

In addition to $y^{H}(k)$, the HDCS gets a force/torque vector through the user interface, which consists of a force-torque sensor. It should be noticed that the HDES can not distinguish exactly between a user's intervention and an interaction of the manipulator with the environment.

In the DCS the manipulator (CTS) will be controlled by a position, velocity and/or force scheme. The position of the manipulator is given by the vector $y(t)$ that consists of the six encoder values of the joints. The output of the DCS is the vector $\boldsymbol{u}(t)$ that consists of the six values for the DAC for each joint motor.

\section{Image-Based Control}

None of the currently known robotics systems are directly using the medical image data like CT or MRI. Most of the systems derive trajectories or target points from the image data during a preoperative planning process. Therefore, an intraoperativ change of treatment parameters is not possible. In case of small changes or misinterpretation of the image data by the surgeon the robot supported treatment has to be interrupted. This may be acceptable as far as a change to conventional treatment methods is possible, but new treatment methods introduced by the advantages of robotics systems eventually do not allow a change. 
To avoid these difficulties the described concept of the robot application for maxillofacial surgery provides the real-time access to image data from a predefined region of interest. That means on one hand the surgeon has the full image information plus preoperative defined abstractions during the whole intervention (paths, targets, etc.). On the other hand the robot controller uses the image data as normal sensor data.

In the application of the robotics system for the placement of the implants the image data are used to give the surgeon a direct feedback where the optimal drilling positions can be found. A slice through the images is computed which starts at the current tool position in the images and has the orientation of the working direction of the drilling machine. Using an approximated interval of hounsfield units for the bone the thickness of the skull bone can be determined.
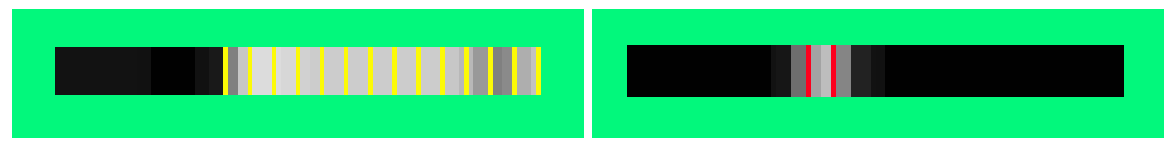

Fig. 4: Slices through the CT images in the working direction of the drill. The thickness of the bone is determined in this section using a fixed threshold. The vertical lines indicate the thickness of the first bone segment. The distance between the lines is $1 \mathrm{~mm}$. The segment starts at the drill position (left side of each segment) and is $2 \mathrm{~cm}$ long. In the left segment enough bone is below the drill. In the right segment the bone is to thin.

A virtual wall is erected in front of bony structures if the bone structure is thinner than the currently used depth of the drill ( 3 or $4 \mathrm{~mm}$ ). Therefore the robot's movements are stopped if the TCP approaches the bone. In this way the surgeon can „feel“" where the placement of the implants is possible through a haptic interface.

In this case the Human Discrete Event System (HDES) computes the velocity of the system based on the desired velocity, the distance to the surface of the skull bone $\operatorname{dist}\left({ }^{I} \boldsymbol{p}_{T C P}\right)$, and its computed thickness thick $\left({ }^{I} \boldsymbol{p}_{T C P}\right)$ :

$$
\boldsymbol{u}^{H}(k)=\left({ }^{I} \dot{\boldsymbol{p}}_{T C P}\right)=O\left(\boldsymbol{F}_{u}, \operatorname{dist}\left({ }^{I} \boldsymbol{p}_{T C P}\right), \operatorname{thick}\left({ }^{I} \boldsymbol{p}_{T C P}\right)\right)
$$

The desired velocity is computed by the force input of the surgeon $\boldsymbol{F}_{u}$. This force control scheme has been described in [13]. A main influence on the successful application of the image-based control of a robotics system is the accuracy of the spatial mapping of the real patient to the coordinate system of the robot. The accuracy depends on two transformations:

$$
\text { pat }_{\boldsymbol{T}}^{\stackrel{f}{\longrightarrow}}{ }^{c t} \boldsymbol{T} \stackrel{g}{\longrightarrow}{ }^{o o b} \boldsymbol{T}
$$

$f$ transforms the real patient into an image coordinate system. The transformation contains quantification errors and errors due to the movement of the patient. For CTs the error depends on the slice distance and the window size of the image. An usual voxel size is $2.0 \times 0.5 \times 0.5 \mathrm{~mm}^{3} . g$ is the transformation between the image coordinate system and the base coordinate system of the manipulator. This transformation is computed after the registration. Inherent errors in $g$ arise from non-rigid fixation of the 
patient, movements of soft tissue, the type of markers, and measurement errors. The amount of errors in $g$ varies between a few tenths and a few millimeters. The image data part of the robot controller display is shown in Fig. 5. The maximum size of the region of interest is about 150 pixel $\times 150$ pixel $\times 30$ slices. Using such a RoI size the image data will be updated with a frequency of $10 \mathrm{~Hz}$, which is the frequency of the navigation system.
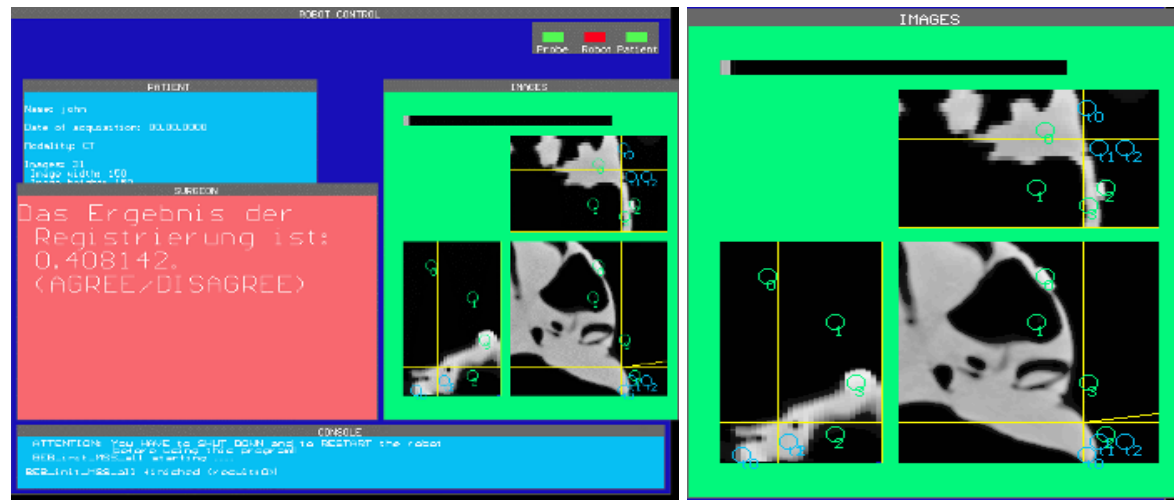

Fig. 5: Screen of the robot controller (left) and a detail of the real-time displaying window with CT data from a plastic scull (right). From the original CT image (in the right window) coronal and sagittal slices (left and above the original slice) and a slice in the working direction (left top) is calculated and the current tool position is superimposed.

\section{Conclusions}

In the paper new concepts for an interactively usable robotics system have been described. Advantages of this concept are the better acceptance by the surgeons, the improved safety of the procedure, and the easy adaptation of the treatment to the actual requirements.

The usability of the interactive tool control has been approved be surgeons in experimental operations at phantoms. The control has been accepted and the first experiments have shown the overall system's suitability for the implant fixation. Up to now, experiments have been carried out with stereolithographic models of a head, where a superconstruction with a preoperative known size has been mounted at the implants. The measured thickness of the bone in CT images depends to a great extend on the chosen window of houndsfield values. Depending on the window the measured bone thickness differs in a great extend. Further research work has to be done to determine an optimal window for measuring the bone thickness and inaccuracies caused by individual differences of the bone structure have to be determined. 


\section{Acknowledgment}

This research work has been performed at the Department for Maxillofacial Surgery, Prof. Dr. Dr. Juergen Bier, within the Surgical Robotics Lab, Prof. Dr. Tim C. Lueth, Medical Faculty Charité, Humboldt-University Berlin. The work has been supported by the Deutsche Forschungsgemeinschaft with the Graduiertenkolleg Temperaturabhängige Effekte (granted to Prof. Dr. Dr. h.c. R. Felix, Prof. Dr. N. Hosten) and by the Real-Time Control Group, Prof. Dr.-Ing. Guenter Hommel, of the Technical University Berlin. Parts of the research have been supported financially by the Deutsche Krebshilfe (granted to Prof. Dr. Dr. J. Bier, PD Dr. P. Wust) and the Berliner Sparkassenstiftung Medizin (granted to Prof. Dr. T. Lueth, Dr. Dr. Ernst Heissler, Prof. Dr. Dr. Berthold Hell). Special thanks to the companies Elekta, Metalor and Philips for their support of the project. We would like also to thank Thomas Hölper, Edgar Schüle, Dr. h.c. Hervé Druais, Dr.-Ing. Armin Freybott, and W. Scholz. Their personal engagement was the basis for this challenging research.

\section{References}

1. Kwoh, Y. S. et al.: A robot with improved absolute positioning accuracy for CT guided stereotactic surgery. Trans. on Biomedical Engineering, Vol. 35, No. 2 (1988) 153-161

2. Lavallée, S.: A new system for computer assisted neurosurgery. Proc. 11th IEEE Engineering in Medicin and Biology Conf., Seatle, November, (1989) 926-927

3. Glauser, D. et al.: Conception of a robot dedicated to neurosurgical operations. Proc. 5th Int. Conf. on Advanced Robotics, Pisa, (1991) 888-892

4. Watanabe, E. et al.: Open surgery assisted by neuronavigator, a stereotactic articulated sensitive arm. Neurosurgery, Vol. 28, No. 6 (1991) 792-800

5. Lavallee, S.: Registration for Computer-Integrated Surgery: Methodology, State of the Art. In Taylor, R. H., S. Lavallee, G. C. Burdea, R. Mösges (eds.), Computer-Integrated Surgery, Technology and clinical Applications, MIT Press, (1996) 77-98

6. Taylor, R. H. et al.: An Image-Directed Robotic System for Precise Orthopaedic Surgery. IEEE Trans. on Robotics and Automation, Vol.10, No.3 (1994) 261-275

7. Ho, S. C. et al.: Force control for robotic surgery. ICAR IEEE Int'l. Conf. on Advanced Robotics, (1995) 21-31

8. Jacobs L. K., V. Shayani, J. M. Sackier: Determination of the learning curve of the AESOP robot. Surg Endosc, Jan; 11(1) (1997) 54-55

9. Cutting, C., Bookstein, F., Taylor, R.: Applications of simulation, morphometrics and robotics in craniofacial surgery. In Taylor, R. H., S. Lavallee, G. C. Burdea, R. Mösges (eds.), Computer-integrated surgery: technology and clinical applications, MIT Press, (1996) 641662

10.Bohner, P. et al.: Operation planning in cranio-maxillo-facial surgery. Medicine Meets Virtual Reality 4 (MMVR4'96), San Diego, California, (1996)

11.Brånemark System ${ }^{\circledast}$ product information for fixure placement - surgical procedure - craniofacial rehabilitation

12.Lueth, T.C. et al.: A Surgical Robot System for Maxillofacial Surgery. IEEE Int. Conf. on Industrial Electronics, Control, and Instrumentation (IECON), Aachen, Germany, Aug. 31Sep. 4, (1998) 2470-2475

13.Hein, A. and T.C. Lueth: Robot Control in Maxillofacial Surgery. Sixth International Symposium on Experimental Robotics, Sydney, Australia , March 26-28, (1999) in print 